\title{
ESTUDO ECONÔMICO DO CULTIVO DE SOJA COM SAFRINHA DE MILHO E GIRASSOL
}

\author{
Stefânia Alves Peixoto ${ }^{1}$, Francielle Wanderley Ribeiro' ${ }^{1}$, Carolina Candida \\ Rodrigues $^{1}$, Andrécia Cósmem da Silva ${ }^{2}$, Matheus da Silva Araujo ${ }^{3}$ \\ ${ }^{1}$ Discente do Curso de Agronomia da Universidade Estadual de Goiás, Campus \\ Ipameri, Ipameri, GO, Brasil. (fran3wanderley@gmail.com) \\ ${ }^{2}$ Professora Mestre da Universidade Estadual de Goiás, Campus Ipameri, Ipameri, \\ GO, Brasil \\ ${ }^{3}$ Mestre em Ciências Florestais da Universidade de Brasília, Campus Darcy Ribeiro, \\ Brasília, DF, Brasil
}

Recebido em: 06/04/2018 - Aprovado em: 10/06/2018 - Publicado em: 20/06/2018 DOI: 10.18677/EnciBio_2018A47

\begin{abstract}
RESUMO
O objetivo desse trabalho foi avaliar a viabilidade econômica da produção de soja em 150ha na safra e o cultivo de milho e girassol em 75ha cada na safrinha, em uma propriedade rural no município de Anápolis, Goiás. O estudo de viabilidade analisou os indicadores Valor Presente Líquido (VPL), Relação Benefício/Custo (RB/C) e Payback atualizado. Foi também realizada a análise de sensibilidade para averiguar a influência da queda ou aumento da taxa de desconto sobre o fluxo de caixa. Para a análise de sensibilidade fez-se a variação no cenário da taxa de desconto sobre o fluxo de caixa utilizando o cenário real que é de $7 \%$ a.a., o pessimista de $8 \%$ a.a. e o otimista de $6 \%$ a.a. A análise de viabilidade econômica apresentou taxa de desconto de $7 \%$ a.a. no decorrer dos 5 anos de análise $V P L=R \$ 167.895,72, R B / C=1,04$ e PayBack atualizado=no quarto ano. Com os resultados obtidos foi possível concluir que o projeto é viável, porém é um cenário de risco por apresentar o retorno dos investimentos apenas no quarto ano. A análise de sensibilidade pareceu viável para todas as taxas de desconto calculadas. O plantio de soja com sucessão de milho e girassol na safrinha apresentou viabilidade econômica para horizonte de planejamento de cinco anos, sendo uma boa opção de investimento para os produtores da região.
\end{abstract}

PALAVRAS-CHAVE: grandes culturas, indicadores econômicos, levantamento de custos.

\section{ECONOMIC STUDY OF SOYBEAN CULTIVATION WITH CORN AND SUNFLOWER CROPS}

\begin{abstract}
The objective of this work was to evaluate the economic viability of soybean production in 150ha and harvest the corn and sunflower cultivation in 75ha each in the off-season, in a country estate in the municipality of Anápolis, Goiás. The feasibility study examined indicators net present value (NPV), benefit/cost (RB/C) and Payback updated. It was also conducted sensitivity analysis to determine the influence of fall or rise in the discount rate on the cash flow. For the sensitivity
\end{abstract}


analysis has been the change in the discount rate on cash flow using the actual scenario of $7 \%$ p.a., the optimist of $8 \%$ p.a. and the pessimist of $6 \%$ p.a. The economic feasibility analysis presented a discount rate of $7 \%$ per year over the 5 years of analysis $\mathrm{VPL}=\mathrm{R} \$ 167.895,72, \mathrm{RB} / \mathrm{C}=1.04$ and PayBack updated $=$ in the fourth grade. With the obtained results it was possible to conclude that the project is feasible, but it is a risk scenario by presenting the return on investment only in the fourth year. The sensitivity analysis seemed viable for all discount rates calculated. The planting soybeans with corn and sunflower succession in the off-season has presented economic viability for five-year planning horizon, being a good investment option for producers in the region.

KEYWORDS: field crops, economic indicators, survey costs.

\section{INTRODUÇÃO}

O agronegócio é de fundamental importância para a economia brasileira, pois participa da geração de renda e emprego, conferindo ao país um papel singular no comércio mundial (ASSAD et al., 2012). Este setor pode ser entendido como a cadeia produtiva que abrange desde a fabricação de insumos, passando pela produção nos estabelecimentos agropecuários, pela sua transformação, até o seu consumo (JESUS; REGÔ, 2014).

A agricultura possibilitou o crescimento econômico bem como elevou a relevância do Brasil no exterior, visto que o país é um dos maiores produtores e exportadores de grãos do mundo, principalmente quando se refere à produtos alimentícios. Portanto, é decisivo que para o bom andamento deste setor, esteja vinculado ao desenvolvimento econômico, social e sustentável do Brasil (ZANELLA; LEISMANN, 2017).

No estado de Goiás, o setor agrícola ganhou forte fervor em meados da década de setenta, quando o Estado agiu de forma categórica na ocupação do Cerrado e na sua integração às atividades produtivas (GUIMARÃES et al., 2011). Atualmente a base da economia goiana constitui-se na exploração agropecuária, produção de grãos e atividades de bovinocultura.

Segundo o Instituto Brasileiro de Geografia e estatística - IBGE (2017), a produção de grãos no ano de 2017 da região centro-oeste ficou em 94.980.255 toneladas, e em Goiás a produção média foi de 22.541.710 toneladas. Com esse volume o estado ficou em quarto lugar no ranking nacional, ficando atrás apenas dos estados de Mato Grosso do Sul, Paraná e Rio Grande do Sul.

A soja é uma cultura agrícola brasileira que mais cresceu nas últimas três décadas e equivale a $49 \%$ da área plantada em grãos do país. A expansão da produtividade está associada aos avanços tecnológicos, ao manejo e a eficiência dos produtores. O grão é componente fundamental na fabricação de rações animais, e seu uso na alimentação humana encontra-se cada vez mais em crescimento (MARCON et al., 2017).

O milho é uma das culturas mais importantes mundialmente, seja do ponto de vista econômico, seja do ponto de vista social. A utilização do milho é ampla, abrangendo seu emprego como alimento, assim como para uso industrial e energético, e seu destino é principalmente a produção de rações para uso animal (ESALQ, 2015). O cultivo do milho é tradicionalmente praticado na safrinha de outono-inverno sob condições de sequeiro, sendo semeado em sucessão à soja cultivada no verão (RICHETTI et al., 2015).

A cultura do girassol está em destaque na economia mundial, por apresentar um elevado consumo para a produção de óleo, durante a safra 2015/2016, teve um 
aumento de $3,1 \%$ na utilização dos grãos, passando para 15,8 milhões de toneladas (CONAB, 2017). Além de ser uma das principais matérias primas para a produção de biodiesel, a cultura pode ser utilizada nos sistemas de rotação e sucessão nos sistemas produtivos (BORSUK et al., 2011).

A compreensão do custo de produção representa uma variável expressiva para a análise econômica e para manter a sustentabilidade da propriedade agrícola. Além de conhecer o custo de produção das atividades econômicas é necessário que o produtor acompanhe o mercado para conhecer os preços cometidos e estabelecer o momento de vender sua produção (RICHETTI; CECCON, 2014).

O acompanhamento financeiro de sistemas de produção frequentemente empregados no setor produtivo, tem propósito de subsidiar dados para tomadas de decisão e verificar a rentabilidade de projetos a serem implantados, das atividades do agronegócio (CARVALHO et al., 2016). Assim sendo, é possível realizar análise de viabilidade econômica e financeira das atividades desenvolvidas, buscando analisar quais são os benefícios esperados em tal investimento para compará-lo com os investimentos e custos do mesmo, assim verificando sua viabilidade de implementação (ZAGO et al., 2009). Sendo relevante a importância da análise de custos, o trabalho teve como objetivo avaliar a viabilidade econômica da implantação da soja na safra e o cultivo de milho e girassol na safrinha, em uma propriedade rural no município de Anápolis, Goiás.

\section{MATERIAL E MÉTODOS}

O experimento foi realizado em uma propriedade no município de AnápolisGO, região do planalto central com as coordenadas, Latitude: $16^{\circ} 16^{\prime} 36.94^{\prime \prime} S$, Longitude: $48^{\circ} 51^{\prime} 56.49^{\prime \prime} \mathrm{W}$ e altitude de $1042 \mathrm{~m}$, clima da região segundo a classificação de Köppen é definido como tropical com estação seca de inverno (AW), com duas estações bem definidas, sendo verão chuvoso e quente, inverno frio e seco, apresentando uma temperatura média na região de $20^{\circ} \mathrm{C}$ (ALVARES et al. 2013).

A propriedade possui área total de 199ha, destes 49ha estão inseridos, reserva legal, área de preservação permanente e benfeitorias dentro da propriedade. Para a análise econômica foram utilizados 150 ha, sendo cultivada a soja na safra, e milho e girassol na safrinha em 75ha cada.

Para a análise do ambiente, foram observadas as variáveis internas e externas que possam impactar nos resultados econômicos. Foram levantados os pontos fracos, fortes, ameaças e oportunidades com vistoria in loco a propriedade rural, como quadro de funcionários, patrimônio existente e a infra-estrutura, para assim proceder a coleta de dados e proceder à análise econômica do estudo. Estudos de mercado também foram realizados para verificar a demanda e oferta para as culturas em questão.

O solo da área é classificado como Latossolo Vermelho Distrófico, com horizontes A de textura arenosa e horizonte diagnóstico subsuperficial B latossólico de textura franco-arenosa. São profundos e porosos ou muito porosos, apresentam condições adequadas para um bom desenvolvimento radicular em profundidade (EMBRAPA, 2013).

Para estimativa do plantio das culturas foi utilizado o sistema plantio direto, em solo que já havia passado por processo de dessecação. O cultivo da soja (Glycine max L. Merr) e posteriores cálculos utilizou-se a cultivar precoce, com ciclo de 93 a 103 dias para colheita, densidade de 15 sementes por metro linear, com espaçamento de $0,50 \mathrm{~m}$, gerando um total de 320.000 plantas.ha-1. 
No cultivo do milho (Zea mays L.) utilizou-se a variedade de milho híbrido precoce, com ciclo de 125 a 140 dias e com semeadura logo após a colheita da soja, sendo esta com espaçamento de $0,50 \mathrm{~m}$, com 3sementes.m, totalizando 60.000 plantas.ha ${ }^{-1}$. Para cultivo do girassol (Helianthus annuus), realizou o plantio também com a cultivar precoce, com ciclo de 125 a 140 dias. A semeadura ocorre logo após a colheita da soja, espaçamento de $0,50 \mathrm{~m}, 7$ sementes.m, gerando um total de 45.000 a 60.000 plantas. ha ${ }^{-1}$.

Foram estimados os cálculos para os possíveis tratos culturais da área como aplicação de inseticidas fungicidas, herbicida, e mão de obra necessária para a execução de todas as atividades. Atribuindo assim, melhores resultados na hora da colheita.

De acordo com o manejo proposto para as culturas, e médias de produtividade da região, estimou-se para a soja 60 sacas.ha $^{-1}$, o milho com 112 sacas.ha $^{-1}$ e o girassol com 34sacas.ha ${ }^{-1}$, para a comercialização considerou-se o preço de mercado da região produtora para cada cultura, sendo $R \$ 63,00, R \$ 27,00$ e $\mathrm{R} \$ 61,92$ respectivamente.

A partir dos valores apresentados pelo fluxo de caixa, receitas e despesas apuradas, utilizou-se para análise de viabilidade econômica os seguintes indicadores: o Valor Presente Líquido (VPL), a Razão Benefício Custo (RB/C) e o PayBack, realizando também a análise de sensibilidade.

O Valor Presente Líquido (VPL) calculado pela Equação 1 consiste em avaliar os valores de todas as entradas e saídas de caixa. É a soma de todos os capitais provenientes das receitas produzidas, diminuído da soma das despesas cometidas do empreendimento, atualizados a partir da taxa de desconto do capital, adotada pelo investimento. O investimento será considerado viável se o VPL for positivo sendo mais atrativo quanto maior for seu valor (LUCENA et al., 2016).

$$
V P L=\sum_{\mathrm{t}=0}^{\mathrm{n}} \frac{F C_{t}}{(1+i)^{t}}
$$

em que:

$\mathrm{FC}=$ fluxo de caixa do período $\mathrm{n}$

$I=$ taxa de desconto

$\mathrm{T}=$ período

A Relação Benefício Custo (Relação B/C) calculada pela Equação 2 é um indicador de eficácia econômica financeira e faz referência ao retorno dos investimentos a partir da comparação entre receitas e despesas atualizadas à taxa de desconto, ou seja, é quanto se espera ganhar para cada unidade de capital investido (ARAÚJO et al., 2015). Se a relação B/C for $>1$ o projeto é considerado viável, resultados $=1$, são considerados de risco, mas ainda realizáveis, $<1$ o projeto não se torna viável e considerado de alto risco.

Relação $B / C=\frac{\sum_{j=0}^{n} R_{j} /(1+i)^{j}}{\sum_{j=0}^{n} c_{j} /(1+i)^{i}}$

em que:

$\mathrm{i}=$ Taxa de desconto

$R \mathrm{j}=$ Receitas oriundas do projeto no ano $\mathrm{j}$

$\mathrm{C} \mathrm{j}=$ Custos do projeto no ano $\mathrm{j}$

$\mathrm{n}=$ Vida útil do projeto 
O PayBack atualizado (Payback) determinado por meio da Equação 3 têm como princípio, considerar a dimensão do tempo de capital, a partir da atualização do fluxo líquido, sob uma determinada taxa de desconto (PARAENSE et al., 2013).

Payback $=\sum_{i=0}^{k} \frac{F i}{(1+j)^{i}} \geq 0$ e $\sum_{i=0}^{k-1} \frac{F i}{(1+j)^{i}}<0$

em que,

$\mathrm{k}=$ Total de períodos do projeto

$\mathrm{Fi}=$ Fluxo de caixa no ano $\mathrm{i}$

$\mathrm{j}=$ Taxa de juros considerada

$\mathrm{i}$ = Número de períodos

E a análise de sensibilidade torna possível a verificação do ponto de estabilidade, ou seja, o equilíbrio do projeto (RICHETTI, 2016). As variações foram feitas em cima da taxa de desconto, sendo $7 \%$ ao a.a. no cenário real (TJLP, 2017), levando para o cenário otimista a taxa, calculada à $6 \%$ a.a. e caso ocorra um aumento da taxa, criando um cenário pessimista, a taxa foi de $8 \%$ a.a. Objetivou-se com a análise de sensibilidade, projetar possíveis panoramas, que pudesse demonstrar e contextualizar algumas das possíveis situações que o produtor rural pudesse se deparar durante a sua atividade. Os dados foram obtidos durante a safra 2016/2017, com um período de cinco anos para análise. As informações foram tabuladas e trabalhadas com a utilização de planilhas eletrônicas do programa Microsoft Excel ${ }^{\circledR}$.

\section{RESULTADOS E DISCUSSÃO}

O conhecimento dos custos de produção de qualquer empreendimento econômico adota papel importante no processo da tomada de decisão sobre a atividade. Diante disso foram levantados os custos de cada cultura, onde a cultura da soja obteve um custo total de $\mathrm{R} \$ 2.726,91$ e o maior investimento ocorreu durante o pré-plantio $\mathrm{R} \$ 656,06$ representado por $24,06 \%$ dos custos, sendo esse valor resultado de operações do preparo do solo, estando inclusos os processos de correção do solo, e dessecação de ervas daninhas. Para as culturas do milho e do girassol pode-se observar que os maiores investimentos ocorreram durante o plantio, representando $R \$ 803,25$ com participação de $32,56 \%$ e $R \$ 577,94$, sendo $31,29 \%$ dos totais de $\mathrm{R} \$ 2.466,62$ e $\mathrm{R} \$ 1.847,01$, respectivamente. Para a cultura do milho, esse resultado é referente principalmente à compra das sementes, por possuir tecnologia para serem resistentes às principais lagartas do milho e também ao uso de herbicidas, que reduz os custos com insumos. O girassol por ser uma cultura exigente em boro apresentou durante os custos de plantio um maior investimento em fertilizantes.

Para a cultura da soja (Tabela 1) o plantio representou o segundo lugar em relação aos custos, totalizando $\mathrm{R} \$ 652,67$ cerca de $23,93 \%$ do total, os custos com plantio estão subdivididos em maquinários, mão de obra, fertilizantes, sementes, inoculantes e defensivos utilizados no tratamento das sementes. Outro fator que pesa é a condução da lavoura em torno de $\mathrm{R} \$ 606,64$ este valor representando $22,25 \%$ do total o qual está distribuído em operações mecânicas, mão de obra e insumos. Por fim, tem-se custos menos expressivo, que compõem o investimento, como os custos com colheita $(8,77 \%)$, pós colheita $(2,98 \%)$, finanças $(6,78 \%)$ e o outros custos $(11,23 \%)$, que apresenta-se um pouco maior, pois é calculada de 
acordo com o tempo de vida útil das máquinas utilizadas para conduzir a lavoura em todos os processos.

TABELA 1. Custo de produção de 1 hectare de soja no estado de Goiás, 2016/2017. Soja Transgênica RR

\begin{tabular}{ccc}
\hline Componentes de custo & Valor $\left(\mathrm{R} \$ \cdot \mathrm{ha}^{-1}\right)$ & Percentual $(\%)$ \\
\hline Custo do pré-plantio & $\mathrm{R} \$ 656,06$ & $24,06 \%$ \\
Custo do plantio & $\mathrm{R} \$ 652,67$ & $23,93 \%$ \\
Custo da condução da & $\mathrm{R} \$ 606,64$ & $22,25 \%$ \\
lavoura & & \\
Custo da colheita & $\mathrm{R} \$ 239,15$ & $8,77 \%$ \\
Custo da pós-colheita & $\mathrm{R} \$ 81,20$ & $2,98 \%$ \\
Custo de despesas & $\mathrm{R} \$ 184,96$ & $6,78 \%$ \\
$\quad$ financeiras & $\mathrm{R} \$ 306,23$ & $11,23 \%$ \\
Outros custos & $\mathrm{R} \$ 2.726,91$ & $100 \%$ \\
\hline Total de custos &
\end{tabular}

O custo de produção de soja obteve um total de $\mathrm{R} \$ 2.726,91$. ha $^{-1}$, em estudo desenvolvido por Richetti (2012), os valores apresentados diferem do presente estudo, apresentando um custo de $\mathrm{R} \$ 1.836,66$. ha $^{-1}$, porém apresentam similaridade quanto aos gastos com defensivos para a condução da lavoura, cerca de $20 \%$ do total. Tal diferença entre valores se justifica pela diferença dos manejos utilizados em diferentes regiões, principalmente quanto à necessidade de insumos durante a condução da lavoura, a variação dos valores de uma região para outra e o aumento do valor de comercialização dos insumos no decorrer dos anos.

O milho safrinha obteve o segundo maior custo com a condução da lavoura com $\mathrm{R} \$ 230,67$, que equivale a $23,38 \%$ do total. Os outros investimentos apresentaram gastos significativos como: pós-colheita $(16,98 \%)$, outros custos $(10,20 \%)$, colheita $(9,35 \%)$, finanças $(4,63 \%)$ e o valor de menor expressão foi com o pré-plantio, por ser em sucessão com a cultura da soja seu custo ficou em apenas 2,90\% (Tabela 2).

TABELA 2. Custo de produção de 1 hectare de milho no estado de Goiás, safra $2016 / 2017$.

\begin{tabular}{ccc}
\hline & Milho Safrinha & \\
\hline Componentes de custo & Valor $\left(\mathrm{R} \$ . h \mathrm{~h}^{-1}\right)$ & Percentual $(\%)$ \\
\hline Custo do pré-plantio & $\mathrm{R} \$ 71,42$ & $2,90 \%$ \\
Custo do plantio & $\mathrm{R} \$ 803,25$ & $32,56 \%$ \\
Custo da condução da & $\mathrm{R} \$ 576,81$ & $23,38 \%$ \\
$\quad$ lavoura & $\mathrm{R} \$ 230,67$ & \\
Custo da colheita & $\mathrm{R} \$ 418,80$ & $9,35 \%$ \\
Custo da pós-colheita & $\mathrm{R} \$ 114,15$ & $16,98 \%$ \\
Custo de despesas & & $4,63 \%$ \\
financeiras & $\mathrm{R} \$ 251,52$ & $10,20 \%$ \\
Outros custos & $\mathrm{R} \$ 2.466,62$ & $100 \%$ \\
\hline Total de custos & &
\end{tabular}

Em estudo apresentado por Richetti et al. (2017) sobre o cultivo de milho safrinha, obtiveram resultados semelhantes ao desenvolvido neste trabalho, no qual o total foi de $\mathrm{R} \$ 2.489,88$. ha ${ }^{-1}$ e os insumos geraram os maiores gastos do projeto ENCICLOPÉDIA BIOSFERA, Centro Científico Conhecer - Goiânia, v.15 n.27; p. 2592018 
totalizando $R \$ 1.132,58$. ha $^{-1}$, esse valor é justificado pelo fato do investimento em sementes com tecnologia $\mathrm{Bt}$, que são de alto valor, mas capaz de fornecer uma maior proteção a lavoura, possibilitando assim a redução de custos com defensivos.

Com o plantio do girassol o custo de maior relevância foi o valor de condução da lavoura, obtendo $\mathrm{R} \$ 557,05$ que consiste em $30,16 \%$ do total. Os gastos restantes ficaram divididos entre: colheita $(12,49 \%)$, pós-colheita $(9,10 \%)$, outros custos $(8,83 \%)$, pré-plantio $(4,59 \%)$ e finanças $(3,55 \%)$ (Tabela 3$)$.

TABELA 3. Custo de produção de 1 hectare de girassol no estado de Goiás, safra $2016 / 2017$.

\begin{tabular}{ccc}
\hline & Girassol Safrinha & \\
\hline Componentes de custo & Valor $\left(\mathrm{R} \$ . h \mathrm{~h}^{-1}\right)$ & Percentual (\%) \\
\hline Custo do pré-plantio & $\mathrm{R} \$ 84,82$ & $4,59 \%$ \\
Custo do plantio & $\mathrm{R} \$ 577,94$ & $31,29 \%$ \\
Custo da condução da & $\mathrm{R} \$ 577,05$ & $30,16 \%$ \\
lavoura & & \\
Custo da colheita & $\mathrm{R} \$ 230,67$ & $12,49 \%$ \\
Custo da pós-colheita & $\mathrm{R} \$ 168,00$ & $9,10 \%$ \\
Custo de despesas & $\mathrm{R} \$ 65,51$ & $3,55 \%$ \\
financeiras & $\mathrm{R} \$ 163,02$ & $8,83 \%$ \\
Outros custos & $\mathrm{R} \$ 1.847,01$ & $100 \%$ \\
\hline Total de custos & &
\end{tabular}

As estimativas de custo da produção de girassol obtiveram resultados bem superiores aos resultados encontrados no trabalho de Borsuk et al. (2011), o presente estudo apresentou um custo de $49 \%$ superior. É importante salientar que essa grande diferença ocorre em função das diversificadas formas de manejo, uma vez que, o estudo realizado na cidade de Abelardo Luz - SC foi desenvolvido por agricultores familiares assentados, com apoio de cooperativas o que acarreta na redução dos custos com maquinários e mão de obra, e é válido ressaltar a grande diferença dos valores de comercialização dos insumos e dos serviços prestados alterados de forma significativa no decorrer dos anos, e a melhoria no preço de comercialização está relacionada com a valorização e demanda do grão nas indústrias.

Cabe salientar também que o cultivo do girassol advém em sua maioria na safrinha e é empregado como plantio complementar. Porém ocorrem diversos fatores que resultam em uma baixa produtividade, entre eles: fatores climáticos adversos, falta de assistência técnica e conhecimento da cultura, manejo inadequado do solo e insuficiência no manejo de pragas e doenças.

O fluxo de caixa simula as estimativas de entradas e saídas monetárias do projeto, com a taxa de desconto de $7 \%$ a.a., verifica-se que a receita líquida se tornou positiva no quarto ano apresentando um valor presente líquido de $\mathrm{R} \$ 167.895,72$, isto é, quando as receitas começam a superar os custos para a implantação das culturas (Tabela 4). De acordo com as análises realizadas pelos indicadores econômicos, todos os critérios de avaliação se mostraram economicamente viáveis, demonstrando que a implantação do projeto é viável, porém considerado de risco, em função do baixo valor de B/C $(1,04)$ (Tabela 4), tal fato pode estar relacionado com a comercialização de girassol que é pouco adotada na região, afetando o valor das receitas. 
TABELA 4. Análise Econômica dos critérios avaliados.

\begin{tabular}{cc}
\hline \multicolumn{3}{c}{ Análise econômica } \\
\hline VPL & $167.895,72$ \\
B/C & 1,04 \\
PayBack atualizado & 4 anos \\
\hline
\end{tabular}

Fonte: autores.

Quanto a análise de sensibilidade (Tabela 5), a queda na taxa de desconto de $7 \%$ para $6 \%$ a.a. proporcionou um aumento de $R \$ 23.539,71$ no valor do VPL, já com o aumento da taxa para $8 \%$ a.a. o VPL caiu R $\$ 420,80$ comparando com o cenário real atual de $7 \%$ a.a. Considerando a sensibilidade avaliada no projeto, todos os indicadores apresentaram viáveis, ou seja, VPL resultou em um valor superior a 0 , o RB/C foi superior a 1 . No entanto, apesar do RB/C ter apresentado viabilidade, o mesmo mostrou-se risco, uma vez que, para cada $R \$ 1,00$ investido obteve-se somente o equivalente a $\mathrm{R} \$ 0,4$ de renda líquida.

O Payback demonstrou que o tempo para recuperação do capital investido no projeto foi de quatro anos, este resultado está diretamente relacionado com o grande investimento inicial que deve ser realizado para implantação das culturas, em função da propriedade apresentar carência de infraestrutura e insumos para a implantação das atividades. É importante citar também que no decorrer dos anos esse cenário poderá mudar, aumentando os valores de receitas das culturas.

TABELA 5. Análise de sensibilidade dos critérios avaliados considerando diferentes taxas de juros.

\begin{tabular}{cccc}
\hline \multicolumn{4}{c}{ Análise de Sensibilidade } \\
\hline Taxa de desconto & $\mathrm{VPL}(\mathrm{R} \$)$ & $\mathrm{RB} / \mathrm{C}$ & PayBack atualizado \\
\hline $6 \%^{*}$ & $\mathrm{R} \$ 191.435,43$ & 1,05 & 4 anos \\
$7 \%^{* *}$ & $\mathrm{R} \$ 167.895,72$ & 1,04 & 4 anos \\
$8 \%^{* * *}$ & $\mathrm{R} \$ 142.785,70$ & 1,04 & 5 anos \\
\hline
\end{tabular}

* Cenário otimista; ${ }^{* *}$ Cenário real; ${ }^{* * *}$ Cenário pessimista.

Fonte: autores

Com a análise de sensibilidade, em todos os cenários avaliados, observa-se que os custos para a implantação do projeto, independentemente das taxas de desconto aplicada é viável à condução das culturas, viabilizando um retorno financeiro para o produtor rural.

É importante ressaltar que o projeto pode apresentar diferenças, dependendo da região de plantio, da variedade de semente escolhidas, dos custos dos insumos, e custos de comercialização, que podem fazer com que esses resultados sejam diferentes dos apresentados. Apesar da implantação do projeto apresentar valores dos indicadores favoráveis a implantação, a atividade apresenta risco, uma vez que os valores de VPL e B/C foram baixos. Por fim é importante que os produtores se atentem a otimização de recursos, ao mercado e intempéries que possam causar perdas, o que alteraria os valores dos indicadores. 


\section{CONCLUSÃO}

O plantio de soja com sucessão de milho e girassol na safrinha apresentou viabilidade econômica, no entanto a implantação das três culturas é considerada de risco, em função do baixo retorno econômico e do tempo de retorno dos investimentos.

A implantação da cultura da soja apresenta maiores custos de cultivo seguido da cultura do milho e girassol.

\section{REFERÊNCIAS}

ALVARES, C. A.; STAPE, J. L.; SENTELHAS, P. C.; GONÇALVES, J. L. M.; SPAROVEK, G. Köppen's climate classification map for Brazil. Meteorologische Zeitschrift. v. 22, n. 6, p. 711-728, 2013. Disponível em: <https://doi.org/10.1127/09412948/2013/0507>. doi: 10.1127/0941-2948/2013/0507.

ARAÚJO, E. F.; AGUIAR, A. S.; BARBOSA, M. V. R.; BRITO, W. C.; CORDEIRO, S. A. Rentabilidade de plantios de acácia-australiana e de sistema de integração lavoura-pecuária-floresta no sudoeste do Piauí. Nativa, v. 03, n. 04, p. 268-275, 2015. Disponível em: <http://dx.doi.org/10.14583/2318-7670.v03n04a08>. doi: 10.14583/2318-7670.v03n04a08.

ASSAD, E. D.; MARTINS, S. C.; PINTO, H. P. Sustentabilidade no agronegócio brasileiro. Rio de Janeiro: Fundação Brasileira para o Desenvolvimento Sustentável. 2012.

BORSUK, L. J.; SCHLINDWEN, S. L.; MARTINS, S. R. Viabilidade econômica e fatores limitantes do cultivo de girassol no período da safrinha em Abelardo Luz/SC. Revista Brasileira Agrociência, v.17, n.2-4, p.277-283, 2011. Disponível em: <http://dx.doi.org/10.18539/cast.v17i2.2060>. doi:10.18539/cast.v17i2.2060.

CARVALHO, L. C.; ESPERANCINI, M. S. T.; SANTOS, J. Z.; RIBAS, L. C. Análise comparativa de estimativas de custo de produção e rentabilidade entre sojas RR1 e RR2 pro/ Bt. v. 31, n. 2, p. 186-191, 2016. Disponível em:<http://dx.doi.org/10.17224/EnergAgric.2016v31n2p186-191>. doi: doi.org/10.17224.

CONAB - Companhia Nacional de Abastecimento, Agricultura e Pecuária. A cultura do Girassol. Disponível em: <http://www.conab.gov.br>. Acesso em: 03 mai. 2017.

EMBRAPA - Empresa Brasileira de Pesquisa Agropecuária. Sistema brasileiro de classificação dos solos. 3.ed. Brasília: DF: Embrapa, 2013. 353p.

ESALQ - Escola Superior de Agricultura "Luiz de Queiroz". Visão Agrícola: Milho, Piracicaba-SP. v. $\quad 9, \quad$ n. $13,2015 . \quad$ Disponível em: < http://www.esalq.usp.br/visaoagricola/sites/default/files/Esalq-VA13-Milho.pdf >. Acessado em: 25 de janeiro de 2017.

GUIMARÃES, G. M. A.; RIBEIRO, F. L.; ECHEVERRÍA, A. R. Importância da agricultura familiar para o desenvolvimento sustentável de municípios com predominância do agronegócio. Revista Brasileira de Agropecuária Sustentável, 
v.1, n.2, p.1-11, 2011. Disponível em: <http://dx.doi.org/10.21206/rbas.v1i2.31>. doi:http://dx.doi.org/10.21206/rbas.v1i2.31.

IBGE - Instituto Brasileiro de Geografia e Estatística . Banco de Dados Agregados. Disponível

em: ftp://ftp.ibge.gov.br/Producao_Agricola/Fasciculo_Indicadores_IBGE/estProdAgr_201 702.pdf >. Acessado em: 24 de setembro de 2017.

JESUS, I. R. D.; RÊGO, R. B. Caracterização do agronegócio no mercado acionário brasileiro. Relatórios de pesquisa em engenharia de produção, v.14, n. 3, p. 2937, 2014.

LUCENA, H. D.; PARAENSE, V. C.; MANCEBO, C. H. A. Viabilidade econômica de um sistema agroflorestal com cacau e essências florestais de alto valor comercial em Altamira-PA. Revista de Administração e Negócios da Amazônia, v.8, n.1, p. 73-84, 2016. Disponível em: <https://doi.org/10.18361/2176-8366/rara.v8n1p\%25p>. doi: 10.18361/2176-8366/rara.v8n1p\%25p.

MARCON, E. C., ROMIO, S. C., MACCARI, V. M., KLEIN, C., LÁJUS, C. R. Uso de diferentes fontes de nitrogênio na cultura da soja. Revista Thema, v. 14, n. 2, p. 298-308, 2017. Disponível em: <http://dx.doi.org/10.15536/thema.14.2017.298308.427>. doi: 10.15536/thema.14.2017.298-308.427.

PARAENSE, V. C.; MENDES, F. A. T. FREITAS, A. D. D. Avaliação econômica de sistemas agroflorestais de cacau e mogno na transamazônica: um estudo de caso. Enciclopédia Biosfera, v.9, n.16; p. 2754-2764, 2013. Disponível em:<http://dx.doi.org/10.18361/2176-8366/rara.v8n1p73-84>. doi: 10.18361/21768366/rara.v8n1p73-84.

RICHETTI, A. Viabilidade econômica da cultura da soja na safra 2016/2017, em Mato Grosso do Sul: Embrapa Agropecuária. 2016, 5p. (Comunicado técnico 211).

RICHETTI, A. Viabilidade econômica da cultura da soja na safra 2012/2013, em Mato Grosso do Sul. Dourados: Embrapa Agropecuária. 2012, 9p. (Comunicado técnico 177).

RICHETTI, A.; CECCON, G. Viabilidade econômica da cultura do milho safrinha, 2015, em Mato Grosso do Sul: Embrapa Agropecuária. 2014, 10p. (Comunicado Técnico 196).

RICHETTI, A.; FLUMIGNAN, D. L.; ALMEIDA, A. C. S. Viabilidade Econômica do Milho Safrinha, Sequeiro e Irrigado, na Região Sul de Mato Grosso do Sul: Embrapa Agropecuária. 2015, 13p. (Comunicado técnico 207).

RICHETTI, A; GARCIA, R. A.; FERREIRA, L. E. A. G. Custos de Produção de Soja e Milho Safrinha em Ponta Porã, MS, para a Safra 2016/2017: Embrapa Agropecuária. 2017. 5p. (Comunicado técnico 222). 
TJLP - TAXA DE JUROS DE LONGO PRAZO. 2017. Disponível em: http://idg.receita.fazenda.gov.br/ orientacao/tributaria/pagamentos-eparcelamentos/taxa-de-juros-de-longo-prazo-tjlp/. Acesso em: 08 de ago. 2017.

ZAGO, C. A.; WEISE, A. D.; HORNBURG, R. A. A importância do estudo de viabilidade econômica de projetos nas organizações contemporâneas. In: Congresso Virtual Brasileiro de Administração - CONVIBRA. V. 06, 2009. Disponível em: <http://www.convibra.org/2009/artigos/142_0.pdf>. Acesso em: 14 de abr. 2017.

ZANELLA, T. P.; LEISMANN, E. L. Abordagem da sustentabilidade nas cadeias de commodities do agronegócio brasileiro a partir de sites governamentais. Revista Metropolitana de Sustentabilidade, v. 7, n. 2, p. 6-19, 2017. 